Márcio A. M. Galvão 1 Joel A. Lamounier 2 Elido Bonomo ${ }^{1}$ Margarete S. Tropia ${ }^{1}$ Eliane G. Rezende 1 Simone B. Calic 3 Chequer B. Chamone ${ }^{3}$ Mirtes C. Machado 4 Márcia E. A. Otoni 4 Romário C. Leite 5 Camila Caram 6 Cláudio L. Mafra 7 David H. Walker 8

\section{Rickettsioses emergentes e reemergentes numa região endêmica do Estado de Minas Gerais, Brasil}

\author{
Emerging and reemerging rickettsiosis \\ in an endemic area of Minas Gerais State, Brazil
}

1 Departamento de Nutrição Clínica e Social, Escola de Nutrição, Universidade Federal de Ouro Preto. Ouro Preto, MG 35400-000, Brasil. mgalvao@enut.ufop.br

2 Departamento de Pediatria, Faculdade de Medicina, Universidade Federal de Minas Gerais. Av. Alfredo Balena s/no, Belo Horizonte, MG 30130-100, Brasil.

3 Fundação Ezequiel Dias. Rua Conde Pereira Carneiro 80, Belo Horizonte, $M G$ 30610-150, Brasil.

${ }^{4}$ Diretoria Regional de Saúde Teófilo Otoni. Rua Epaminondas Otoni 849, Teófilo Otoni, $M G$ 39800-000, Brasil.

5 Departamento de Medicina Veterinária Preventiva, Escola de Veterinária, Universidade Federal de Minas Gerais. Av. Antônio Carlos 6627, Belo Horizonte, MG 31270-901, Brasil. 6 Departamento de Estatística, Instituto de Ciências Exatas, Universidade Federal de Minas Gerais. Av. Antônio Carlos 6627, Belo Horizonte, MG 31270-901, Brasil.

7 Departamento de Análises Clínicas, Escola de Farmácia, Universidade Federal de Ouro Preto. Rua Costa Sena 171, Ouro Preto, MG 35400-000, Brasil.

8 Pathology Department, University of Texas Medical Branch. 1116 Keiller Building, 301, University Boulevard, Galveston, TX 77555-0609, U.S.A.
Abstract This article describes a serological survey for rickettsiosis in the county of Novo Cruzeiro, Minas Gerais State, Brazil, in 1998, testing schoolchildren and dogs. Sera included 331 samples from schoolchildren from an endemic area and 142 samples from schoolchildren from a non-endemic area in the county. All children examined were healthy and had not reported clinical symptoms of Brazilian spotted fever prior to the serological survey. Some 35 children in the endemic area were reactive to Rickettsia rickettsii by indirect fluorescent antibody (IFA) with a titer of 1:64, corresponding to 10.6\%. Sera from 73 dogs were tested, showing seroreactivity (IFA 1:64) to Rickettsia rickettsi, Ehrlichia chaffeensis, and Ehrlichia canis in 3 (4.11\%), 11 (15.07\%), and 13 (17.81\%), respectively. The results in schoolchildren and the presence of canine seroreactivity to Ehrlichia species that are potentially pathogenic to humans suggests the risk of transmission of other Rickettsiae in the study area.

Key words Rickettsia rickettsii; Rickettsia Infections; Ehrlichia; Rocky Mountain Spotted Fever

Resumo O trabalho descreve um inquérito sorológico para rickettsioses em escolares e cães de Novo Cruzeiro, Minas Gerais, Brasil, em 1998. Trezentos e trinta e um escolares pertenciam a uma área endêmica e 142 a uma área não endêmica do município. Trinta e nove (10,1\%) soros foram reativos à Reação de Imunofluorescência Indireta (RIFI) para Rickettsia rickettsii no título de 1:64, sendo que dentre esses reativos, 35 eram de estudantes de escolas de área endêmica. Dentre os 73 cães analisados quanto à presença de anticorpos anti R. rickettsii, anti Ehrlichia chaffeensis e anti Ehrlichia canis à RIFI no título de 1:64, 3 (4,11\%), 11 (15,07\%) e 13 (17,81\%) desses animais foram reativos respectivamente aos antígenos testados. Conclui-se que, a sororeatividade para $\mathrm{R}$. rickettsii em indivíduos sadios sem história prévia de febre maculosa brasileira, uma doença marcante por sua alta letalidade, e a presença de sororeatividade para Ehrlichia com potencial patogênico para o homem em cães, nos leva a indagar sobre a transmissão ao homem de outras espécies da família Rickettsiae na área estudada.

Palavras-chave Rickettsia Rickettsii; Rickettsioses; Ehrlichia; Febre Maculosa das Montanhas Rochosas 


\section{Introdução e objetivos}

De todas as doenças que afligiram o homem, as doenças causadas por espécies de bactérias do gênero Rickettsia se situam entre aquelas doenças que mais causaram sofrimento e morte, inclusive para vários pesquisadores pioneiros no diagnóstico e na pesquisa sobre as mesmas (Galvão, 1996).

Historicamente em 1899, Maxcy descreve nos Estados Unidos, as manifestações clínicas da febre das Montanhas Rochosas. No período de 1906 a 1909, Ricketts conseguiu sucesso na transmissão dessa doença para porquinhos da Índia, incriminou o carrapato como vetor, e observou rickettsias em esfregaços preparados a partir de tecidos de carrapatos (Ricketts, 1909).

No ano de 1929, em São Paulo, José Toledo Piza iniciou a distinção da febre maculosa das demais doenças exantemáticas no Brasil, inclusive chegando a demonstrar sua semelhança com a entidade nosológica descrita pelos americanos como Rocky Mountain spotted fever (Piza, 1932).

Durante a Segunda Grande Guerra, surgiram alguns avanços no controle das rickettsioses, como o uso de inseticidas no combate aos vetores e o advento dos antibióticos na década de 40, com resultados importantes no tratamento das rickettsioses (Galvão, 1996).

No que toca à febre maculosa brasileira (FMB), a mesma tem sua ocorrência reconhecida nos Estados de Minas Gerais (Dias \& Martins, 1937; Galvão et al., 1999; Magalhães, 1952), Rio de Janeiro (Gonçalves et al., 1981), Bahia (Mancini, 1983), São Paulo (Melles et al., 1992) e Espírito Santo (Sexton et al., 1993). No Estado de Minas Gerais, a FMB tem ocorrido em áreas onde os residentes estão expostos a habitats infestados por carrapatos. As regiões com maior número de casos no Estado são os vales do Rio Doce, Mucuri e Jequitinhonha, localizados na região nordeste de Minas Gerais (Figura 1). Essas áreas consideradas sob pressão econômica, requerem que indivíduos se aventurem em regiões infestadas por carrapatos em busca de sua sobrevivência. Em 64 casos investigados nessas áreas, foi observada uma taxa de letalidade para FMB de 19\% entre 1993 e 1995 (Galvão, 1996).

Desde que as evidências disponíveis sugerem fortemente, que as rickettsioses podem se constituir numa significante fração não reconhecida dentro dos casos de doença febril a esclarecer nessas populações, a Universidade Federal de Ouro Preto (UFOP) e a Fundação Ezequiel Dias (FUNED) em Minas Gerais, decidiram implantar um programa de vigilância contínua para rickettsioses nessas regiões, incluindo a realização de inquéritos sorológicos em comunidades e em grupos de risco. A intenção desses inquéritos era primeiramente investigar a prevalência sorológica de anticorpos para Rickettsiae nessas populações, afim de que pudéssemos melhor conhecer as espécies de Rickettsia circulantes em nosso meio com potencial patogênico para o homem.

No primeiro inquérito realizado no Município de Novo Cruzeiro, Minas Gerais, no ano de 1998, todos os indivíduos examinados eram sadios, que não reportaram sintomas relacionados à FMB nos últimos três anos anteriores ao inquérito sorológico. A amostragem de soros examinada, ou seja, 141 soros coletados (uma amostra por indivíduo), representava $82,9 \%$ da população total de 170 moradores da localidade de Empoeirado, Município de Novo Cruzeiro. Vinte e seis (18\%) dos soros tinham títulos, na reação de imunofluorescência indireta (RIFI), $\geq 1$ /64 para Rickettsia rickettsii (Galvão et al., 1999). Não houve diferença significante na prevalência de anticorpos entre homens e mulheres e grupos de idade. Nenhum soro possuía anticorpos no título de 1/64 para Rickettsia typhi ou Ehrlichia chaffeensis. Entre os 26 soros que apresentavam anticorpos para $R$. rickettsii, três tinham título de $1 / 128$ e dois de $1 / 256$, os demais $1 / 64$. Vinte e cinco soros entre os 26 reativos à RIFI foram testados também utilizando uma reação mais específica para a presença de anticorpos para $R$. rickettsii, no caso western blotting, com uma diluição de 1:100, revelando anticorpos para antígenos de superfície da $R$. rickettsii, a proteína de superfície de membrana A (rOmpA) e a proteína de superfície de membrana B (rOmpB), em apenas um deles (Galvão et al., 1999). Diminuindo a especificidade com uma diluição de 1:50 para essa mesma reação, 18 soros entre os 19 testados revelaram anticorpos para $R$. rickettsii rOmpA e rOmpB.

Novo Cruzeiro foi o município selecionado para o presente estudo, devido à alta taxa de letalidade (30\%) para FMB durante o período de 1993 a 1995, e à realização nesse município, em uma localidade denominada Empoeirado, do inquérito sorológico humano descrito (Galvão et al., 1999), com resultados altamente significativos no tocante à presença de sorologia positiva para Rickettsiae. Essa localidade apresentava uma população estimada de 170 pessoas, sendo que no ano de 1995, foram registrados seis casos humanos suspeitos de FMB com quatro óbitos, e que dentre os seis, quatro foram confirmados laboratorialmente, três pela RIFI e um pela prova de imunoperoxidase em material de necrópsia (vasos sangüíneos do fígado, estômago e rim).

Este trabalho descreve inquérito realizado em estudantes de quatro escolas de Novo Cruzeiro, e na população canina de Empoeirado, no mesmo 
município, e teve como objetivo geral conhecer as espécies de Rickettsia circulantes, inclusive aquelas com potencial patogênico para o homem.

\section{Material e métodos}

Uma amostra de $10 \mathrm{ml}$ de sangue venoso foi retirada de 473 estudantes do Município de Novo Cruzeiro, no mês de setembro de 1998. Para o propósito deste estudo, o município foi subdividido em duas áreas: uma endêmica (Empoeirado e área adjacente) e uma não endêmica. Da área considerada endêmica foram coletadas 331 amostras de sangue e da não endêmica 142. As amostras analisadas eram provenientes de estudantes de quatro escolas, identificadas neste estudo como A, B, C e D, sendo A e D escolas da área considerada endêmica, e B e C escolas da não endêmica. O soro dessas amostras de sangue foi separado por centrifugação e estocado a $-20 \circ \mathrm{C}$ na University of Texas Medical Branch (UTMB), em Galveston, Texas, Estados Unidos. Na UTMB, os soros foram examinados buscando-se verificar a presença de anticorpos para $R$. rickettsii pela RIFI a uma diluição de 1:64 (Dumler \& Walker, 1994; Kaplan \& Schonberger, 1986; La Scola \& Raoult, 1997; Newhouse et al., 1979). O título mínimo considerado positivo foi 1/64. Foram também coletados soros de 73 cães de Empoeirado (área endêmica), buscando-se através da mesma técnica descrita (RIFI), na mesma diluição e critério de positividade (1/64), a presença de soros positivos para R. rickettsii, Ehrlichia canis e E. chaffeensis.

Dados demográficos, sócio-econômicos e epidemiológicos foram pesquisados no dia da coleta de sangue dos estudantes, através de um questionário aplicado à família, tendo sido obtido dos pais um consentimento por escrito para realização do trabalho e utilização dos dados. Nessas informações estavam incluídas presença de história de doença caracterizada ou por febre e exantema, ou febre e dor de cabeça, admissões hospitalares, doença associada com ataque de carrapato, e história de FMB, todos esses dados pesquisados para os últimos três anos.

O projeto foi submetido e aprovado pelo Comitê de Ética da UFOP.

\section{Resultados e discussão}

Nenhum dos estudantes reportou sintomas clínicos de FMB nos três anos anteriores ao inquérito sorológico. Os soros analisados incluíam 331 amostras de uma área endêmica, e 142 de uma não endêmica do Município de Novo Cru-
Figura 1

Mapa do Estado de Minas Gerais, Brasil, com destaque para as regiões de maior incidência da febre maculosa brasileira.

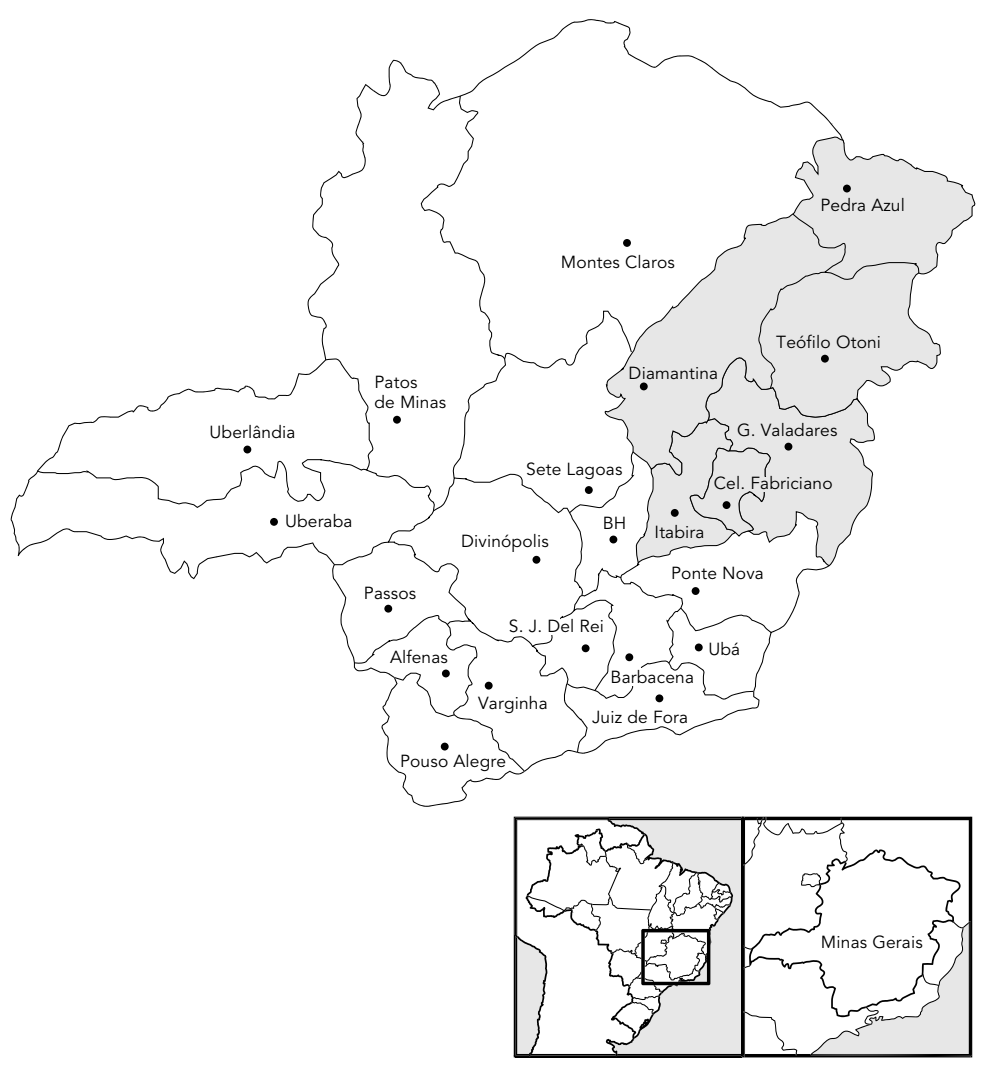

zeiro. Na Figura 2, é possível observar que a prevalência de anticorpos para $R$. rickettsii analisados no Município de Novo Cruzeiro, tem os valores de $12,7 \%, 3,1 \%, 2,3 \%$ e $10,1 \%$ para as escolas A, B, C e D, respectivamente. Nessa mesma figura, podemos observar que 35 estudantes das escolas da área endêmica (escolas A e D) no Município de Novo Cruzeiro, foram sororeativos para $R$. rickettsii correspondendo a $10,6 \%$, e somente quatro estudantes da área não endêmica (escolas B e C) correspondendo a $2,8 \%$, foram sororeativos. No total do município, $10,1 \%$ dos estudantes testados foram sororeativos para a bactéria citada.

Os resultados laboratoriais da sorologia realizada nos cães da área de Empoeirado Novo Cruzeiro, demonstraram a presença de anticorpos anti- $R$. rickettsii, anti E. chaffeensis e anti E. canis com título de 1:64 em 3 (4,11\%), $11(15,07 \%)$ e $13(17,81 \%)$ animais, respectivamente (Figura 3). Dentre os 13 soros reativos pa- 
Figura 2

Sororeatividade à reação de imunofluorescência indireta para anticorpos anti-Rickettsia rickettsii (Título 1:64), em amostras de soro procedentes de estudantes da região de Novo Cruzeiro, Minas Gerais, Brasil.

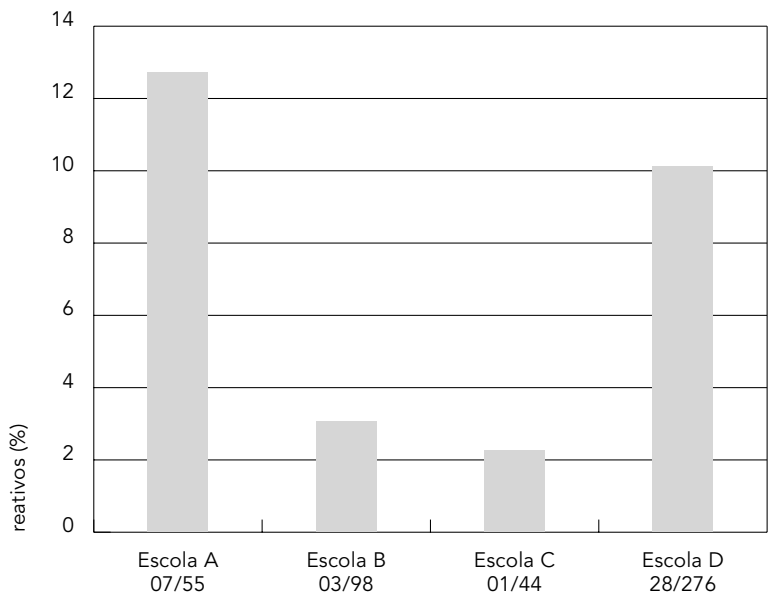

Escolas A e D: escolas de área endêmica; Escolas B e C: escolas de área não endêmica.

Figura 3

Percentual de sororeativos para Rickettsia rickettsii, Ehrlichia canis e Erlichia chaffeensis pela reação de imunofluorescência indireta (1:64) no inquérito sorológico canino. Município de Novo Cruzeiro, Minas Gerais, Brasil, 1998.

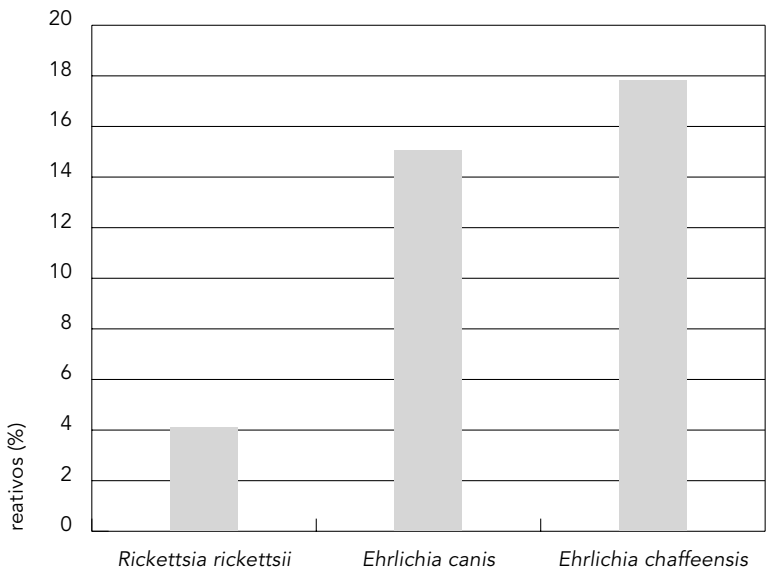

ra E. chaffeensis, encontramos cinco $(38,46 \%)$ soros reativos a 1:64 exclusivamente para $E$. chaffeensis, enquanto oito $(61,54 \%)$ reagiram tanto para E. chaffeensis quanto para E. canis ao mesmo título.

No que toca à sorologia humana, foram realizados e publicados no Brasil desde o início da década de 80 , antecedendo a este trabalho, quatro inquéritos sorológicos para $R$. rickettsii, encontrando-se os seguintes valores de prevalência na população geral à RIFI com o título mínimo de 1/64: 3,17\% (Galvão, 1988); 7,14 \% (Lemos, 1991) ; 2,16\% à RIFI e 4,74\% à Prova de Elisa 1:100 (Galvão, 1996) e 18\% (Galvão et al., 1999). Taylor trabalhando em uma região do Texas nos Estados Unidos, onde ocorrera um surto epidêmico relacionado à febre das Montanhas Rochosas, encontrou entre 352 estudantes de cinco escolas dessa área, $32(9,1 \%)$ reativos à RIFI num título maior ou igual a 1:64 (Taylor, 1985).

O que chama a atenção no presente inquérito realizado em escolares, onde se encontrou $10,1 \%$ de prevalência sorológica, é o fato de todos os estudantes sororeativos terem tido contato com carrapatos, mas não terem tido história de doença febril incapacitante e ou FMB nos três anos que antecederam o inquérito, o que também pôde ser observado por Galvão em estudo na população geral na mesma localidade (Galvão et al., 1999). Como as descrições que temos de FMB é de uma doença de alta letalidade, embora alguns autores questionem a existência de casos oligosintomáticos (Galvão et al., 2000), a principal hipótese que passamos a ter é da existência de outras Rickettsias do mesmo grupo da FMB, patogênicas ou não para o homem, promovendo viragem sorológica cruzada com a $R$. rickettsii, no caso deste trabalho específico, e mesmo talvez em alguns casos das publicações anteriores.

Com relação à presença de sorologia positiva para E. chaffeensis em cães, considerada o agente da ehrlichiose monocítica humana, doença até o momento não descrita no Brasil e que tem como vetor nos Estados Unidos o carrapato da espécie Amblyomma americanum, o fato reveste-se da maior importância devido à descrição primeira no presente artigo, da presença de anticorpos para essa bactéria em cães no Brasil.

Os dados deste levantamento e resultados recentemente encontrados, confirmando a presença de casos com sorologia positiva para Rickettsia felis no Estado de Minas Gerais, Brasil (Raoult et al., 2001), e a presença desta Rickettsia em pulgas Ctenocephalides spp. nesse mesmo estado (Oliveira et al., 2002), demons- 
tra a importância de se investigar mais a presença de outras espécies da família Rickettsiceae com potencial patogênico para o homem, como é o caso de espécies do gênero Ehrlichia, nessa área e em outras regiões do Estado e do Brasil. Visando-se atingir esses objetivos, a implantação de tecnologias avançadas, como a biologia molecular, seria de grande importân-

\section{Referências}

DIAS, E. \& MARTINS, A. V., 1937. Aspectos do typho exanthemático em Minas Gerais. Brasil Médico, 14:431-41.

DUMLER, S. J. \& WALKER, D. H., 1994. Diagnostic tests for Rocky Mountain spotted fever and other rickettsial diseases. Dermatologic Clinics, 12:2536.

GALVÃO, M. A. M., 1988. A Febre Maculosa Brasileira em Minas Gerais e seus Determinantes. Dissertação de Mestrado, Rio de Janeiro: Escola Nacional de Saúde Pública, Fundação Oswaldo Cruz.

GALVÃO, M. A. M., 1996. Febre Maculosa em Minas Gerais: Um Estudo sobre a Distribuição da Doença no Estado e seu Comportamento em Área de Foco Peri-urbano. Tese de Doutorado, Belo Horizonte: Faculdade de Medicina, Universidade Federal de Minas Gerais.

GALVÃO, M. A. M.; CHAMONE, C. B.; CALIC, S. B.; MACHADO, M. C.; OTONI, M. E. A.; DIETZE, R.; MORON, C.; FENG, H. M.; OLANO, J. P. \& WALKER, D. H., 1999. Serologic evidence of spotted fever group Rickettsia in Novo Cruzeiro Municipality - Minas Gerais State - Brazil. In: Rickettsial Diseases at the Turn of the Third Millennium (D. Raoult \& P. Brouqui, ed.), pp. 240-243, Marseille: Elsevier.

GALVÃO, M. A. M.; RIBEIRO, J. G. L. \& MACHADOPINTO, J., 2000. Rickettsioses. In: Doenças Infecciosas na Infância e na Adolescência (E. Tonelli \& L. M. S. Freire, org.), pp. 1517-1529, Rio de Janeiro: Medsi.

GONÇALVES, A. J. R.; LOPES, P. F. A.; MELO, J. C. P.; PEREIRA, A. A.; PINTO, A. M. M. \& LAZERA, M. S., 1981. Rickettsioses: A propósito de quatro casos diagnosticados no Rio de Janeiro de febre maculosa brasileira. Folha Médica, 82:127-134.

KAPLAN, J. E. \& SCHONBERGER, L. B., 1986. The sensitivity of various serologic tests in the diagnosis of Rocky Mountain spotted fever. American Journal of Tropical Medicine and Hygiene, 35:840-844.

LA SCOLA, B. \& RAOULT, D., 1997. Laboratory diagnosis of rickettsioses: Current approaches to diagnosis of old and new rickettsial diseases. Journal of Clinical Microbiology, 35:2715-2727.

LEMOS, E. R. S., 1991. Aspectos Epidemiológicos da Rickettsiose do Grupo da Febre Maculosa em uma Área Endêmica do Estado de Minas Gerais, Brasil. Dissertação de Mestrado, Rio de Janeiro: Instituto Oswaldo Cruz, Fundação Oswaldo Cruz. cia para identificação dos agentes circulantes em vetores como pulgas e carrapatos, e também em pacientes. Para isso, necessita-se estimular a pesquisa em doenças rickettsiais, através da obtenção de recursos específicos e implantação de uma política de formação de recursos humanos nesta área.

MAGALHÃES, O., 1952. Contribuição ao Conhecimento das Doenças do Grupo Exantemático. Rio de Janeiro: Imprensa Nacional.

MANCINI, D. A. P., 1983. A ocorrência de riquetsioses do grupo Rickettsia rickettsii. Revista de Saúde Publica, 17:493-499.

MELLES, H. H.; COLOMBO, S. \& SILVA, M. V., 1992. Spotted fever: Isolation of Rickettsia from a skin biopsy sample. Revista do Instituto de Medicina Tropical de São Paulo, 34:37-41.

NEWHOUSE, V. F.; SHEPARD, C. C.; REDUS, M. D.; TZIANABOS, T. \& McDADE, J. E., 1979. A comparison of complement fixation, indirect fluorescent antibody, and micro agglutination tests for the serological diagnosis of rickettsial diseases. American Journal of Tropical Medicine and Hygiene, 28:387-395.

OLIVEIRA, R. P.; GALVÃO, M. A. M.; MAFRA, C. L.; CHAMONE, C. B.; CALIC, S. C.; SILVA, S. U. \& WALKER, D. H., 2002. Rickettsia felis in Ctenocephalides spp. Fleas, Brazil. Emerging Infectious Diseases, 8:317-319.

PIZA, J. T., 1932. Considerações epidemiológicas e clínicas sobre o Tifo Exantemático de São Paulo. In: Tifo Exantemático de São Paulo (J. T. Piza, J. R. Meyer \& L. Salles-Gomes, org.), pp. 11-119, São Paulo: Sociedade Impressora Paulista.

RAOULT, D.; LA SCOLA, B.; ENEA, M.; FOURNIER, P. E.; ROUX, V.; FENOLLAR, F; GALVÃO, M. A. M. \& DE LAMBALLERIE, X., 2001. A flea-associated Rickettsia pathogenic for humans. Emerging Infectious Diseases, 7:73-81.

RICKETTS, H. T., 1909. A microorganism which apparently has a specific relationship to Rocky Mountain spotted fever. JAMA, 52:379-380.

SEXTON, D. J.; MUNIZ, M.; COREY, G. R.; BREITSCHWERDT, E. B.; HEGARTY, B. C.; DUMLER, J. S.; WALKER, D. H.; PEÇANHA, P. M. \& DIETZE, R., 1993. Brazilian spotted fever in Espírito Santo, Brazil: Description of a focus of infection in a new endemic region. American Journal of Tropical Medical and Hygiene, 49:222-226.

TAYLOR, J. P., 1985. Serological evidence of subclinical Rocky Mountain spotted fever infections in Texas. Journal of Infectious Diseases, 151:367-369.

Recebido em 19 de setembro de 2001

Versão final reapresentada em 1 de março de 2002 Aprovado em 5 de abril de 2002 